\title{
IMPERATIVO, FUTURO E INFINITIVO YUSIVO EN UNA INSCRIPCIÓN DE MITILENE*
}

\author{
Jesús de la Villa \\ Universidad Autónoma de Madrid \\ jesus.delavilla@uam.es
}

\section{RESUMEN}

Sobre la base de un decreto de Mitilene del s. IV a. C., en el que se combinan imperativos, infinitivos y futuros como formas directivas para expresar la orden, se indaga en las posibles diferencias entre estos tres procedimientos alternativos. Tras revisar diversas propuestas de análisis, se concluye que los tres procedimientos son en este texto básicamente intercambiables, pero que se distribuyen según un patrón regular para organizar el texto.

Palabras Clave: Griego antiguo, imperativo, infinitivo, futuro, órdenes, Mitilene.

IMPERATIVE, FUTURE, AND IUSSIVE INFINITIVE IN AN INSCRIPTION FROM MYTILENE

\section{ABSTRACT}

On the basis of a decree from Mytilene, dated in the $4^{\text {th }}$ century a. C., which combines imperatives, infinitives and futures as forms for expressing orders, the author investigates the possible differences between these three alternative procedures. After reviewing various previous proposals, it is concluded that the three procedures are basically interchangeable in this text, but are distributed according to a regular pattern for organizing the text.

KEYwOrDs: Ancient Greek, Imperative, Infinitive, Future, Orders, Mytilene.

\section{EL PROBLEMA}

La diferencia entre el imperativo, el infinitivo yusivo y el futuro yusivo como formas alternativas de transmitir órdenes en griego antiguo es una cuestión extremadamente debatida a lo largo del tiempo en los estudios sobre sintaxis griega (v. infra). El problema está lejos de haber sido resuelto. A nuestro juicio, sigue siendo importante analizar nueva evidencia en documentos y contextos concretos en los que aparezcan usados varios de estos procedimientos al mismo tiempo para transmitir órdenes y prohibiciones. Este tipo de textos, compuestos a la vez, en unas mismas circunstancias y por el mismo autor, proporciona una base inmejorable para establecer diferencias y solapamientos entre tales tipos de expresión. En el presente trabajo analizamos uno de estos documentos, una famosa inscripción de la segunda mitad del s. IV a. C., 
hallada en Mitilene, muchas veces publicada y analizada en aspectos históricos y dialectales, pero que, hasta donde sé, nunca ha sido explotada con el objetivo que me propongo analizar aquí.

\section{ESTADO DE LA CUESTIÓN}

Como se ha dicho, son muchos los estudios específicos o las indicaciones recogidas en manuales sobre la cuestión planteada. A continuación se ofrecen algunas de las propuestas más importantes:

a) Delbrück (1863), Wagner (1891) y Hentze (1902) consideran que el imperativo se utiliza para órdenes concretas, mientras que el infinitivo se usa para órdenes más generales, que no tienen que realizarse de un modo inmediato. Algo semejante parece sugerir, al menos para el infinitivo, sin llegar a asumirlo plenamente Wackernagel (1950, I: 266-267).

b) Kühner - Gerth (1898-1904, vol. II: 23-24) consideran que el infinitivo es la forma más expresiva de dar las órdenes y, por lo tanto, las transmite de un modo más enfático, con más fuerza. El futuro, por su parte (Kühner - Gerth, 1898-1904, vol. I: 176) expresaría más una esperanza que una orden.

c) Brioso (1990), siguiendo a Bers (1984: 181) y Neuberger-Donath (1980), a propósito de un pasaje del Himno a Hermes, recuerda que la secuencia imperativo-infinitivo yusivo es corriente, de tal manera que el infinitivo podría adquirir su valor impresivo como una forma de expresión neutra, cuyo valor se deriva precisamente del contexto directivo. Esto, indirectamente, apuntaría al hecho de que el infinitivo no expresaría nada diferente del imperativo.

d) Hodot (1990) establece una oposición de tipo estructuralista: el infinitivo es la forma neutra de dar las órdenes, el modo más general y puede utilizarse en todos los contextos, mientras que el imperativo tiene un carácter más de previsión concreta. e) García Ramón (2001) se centra en un solo texto, las leyes de Gortina, y, sobre la base de esta larga inscripción, llega a dos conclusiones importantes. En primer lugar, que la diferencia entre todas estas formas de dar órdenes es muy pequeña y que todas pueden utilizarse de forma intercambiable, lo que coincide en parte con lo que puede deducirse de Brioso (1990). En segundo lugar, considera que, si se puede encontrar una diferencia entre el imperativo y el infinitivo es, coincidiendo con Hodot, que el primero es la forma marcada de la oposición y se utiliza para órdenes concretas, precisas, tal y como decían los autores citados en a) más arriba, mientras que el infinitivo se utiliza para órdenes genéricas, a veces sin sujeto claro, o, como término no marcado, en los mismos contextos que el imperativo. En otras palabras, el imperativo es el que no se utilizaría normalmente en contextos de carácter general.

* Este trabajo se ha desarrollado dentro del proyecto "Interacción del léxico y la sintaxis en griego antiguo y latín" (FFI2017-83310-C3-1-P), así como con ayuda adicional del proyecto "Compatibilidad de REGLA con otros instrumentos digitales" (Fundación BBVA, convocatoria de ayuda a las Humanidades Digitales 2018). Agradezco a Araceli Striano las valiosas indicaciones realizadas sobre diversos aspectos del trabajo. 
f) Allan (2010) cambia por completo el punto de vista y pasa a considerar el contexto como la base de la distribución de las diferentes formas de dar órdenes. Según él, el imperativo transmite órdenes fuertes, que dependen de una ley o una regla social ajena al emisor y válidas de un modo independiente del contexto concreto. Por el contrario, el infinitivo es una forma más genérica de dar la orden, depende mucho más del contexto inmediato y no implica una apelación tan directa al receptor de parte del emisor de la orden.

g) En un trabajo anterior sobre un largo texto dialectal tesalio (Villa, 2018) defendí que, al menos en ese texto, de acuerdo con García Ramón, las diferencias gramaticales o semánticas entre las diversas formas de órdenes son muy pequeñas. Sin embargo, sí existía una patrón de distribución de carácter estilístico: en los diferentes párrafos predominaba bien el infinitivo, bien el imperativo, sin una clara distribución de contenido, pero, finalmente, la conclusión de cierre de cada párrafo utilizaba sistemáticamente el imperativo. El futuro, por su parte, se utilizaba de un modo exclusivo en lugar de los otros dos en una segunda parte del texto, ligada a la anterior, pero independiente de ella y sin que pudiera rastrearse una diferencia clara con los contextos anteriores de uso del infinitivo y el imperativo. De todo ello se deducía que las variantes alternativas para dar instrucciones se usan en el texto analizado como una forma de estructuración del discurso, más que atendiendo a eventuales diferencias gramaticales, semánticas o pragmáticas entre ellas.

Como se ve, hay una gran disparidad en el análisis de las diferencias entre estas tres formas alternativas de dar órdenes en griego clásico. Sobre la base de estas propuestas vamos a analizar la inscripción que hemos tomado ahora como objeto de estudio.

\section{EL EDICTO DE LOS EXILIADOS DE MITILENE}

Se trata de una larga inscripción hallada en Mitilene en el siglo XIX y editada muchas veces por su importancia lingüística y, sobre todo, histórica ${ }^{1}$. Recoge los contenidos de un edicto emitido por Alejandro, tradicionalmente datado en torno al año 324 a. C., pero que Heisserer - Hodot (1986) datan, sobre la base de su contenido y las circunstancias históricas, en poco posterior al 334 a. C. En este decreto

${ }^{1}$ En Inscriptiones Graecae XII 26 y Suppl. XII 26 pueden encontrarse dos versiones con diferente reconstrucción de las partes perdidas. Los estudios y conjeturas de reposición para determinados pasajes se han sucedido desde el mismo momento de su hallazgo: cf. Hicks-Hill, 1901:310, para las aportaciones hasta esa fecha. Las conjeturas parciales han sido muchas; un aparato crítico completo puede consultarse en Fazzini (2018). Una revisión de la bibliografía, con un análisis histórico completo se encuentra en Heisserer, 1980: 118-139 (SEG XxX 1041) y en Fazzini (2018). El texto generalmente aceptado hasta ahora es el de Heisserer - Hodot (1986), que reconsideran la totalidad de la inscripción en sus aspectos históricos y textuales. 
se establecen algunas instrucciones con relación al retorno a Lesbos de los exiliados políticos y la restauración del sistema democrático. Todo él está encaminado a lograr una reconciliación social y política y una coexistencia pacífica entre los que se quedaron en la ciudad, en gran medida partidarios de un régimen oligárquico, frente a los que tuvieron que huir y eran partidarios de la democracia y que van regresando.

El texto y la traducción se ofrecen a continuación. Yo sigo la edición de Heisserer - Hodot (1986), salvo donde se indica otra cosa. La inscripción está rota en su parte superior y no sabemos exactamente cuánto se ha perdido. Una parte importante de las reconstrucciones y conjeturas se basan en que el texto está escrito de un modo tal que aparecen muchos paralelismos entre el tratamiento que debe darse a los exiliados retornados y a los ciudadanos que habían permanecido en Mitilene. La comparación de unas frases con otras permite proponer conjeturas probables en muchos casos.

1

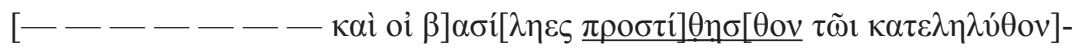

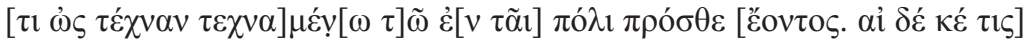

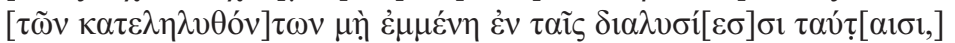

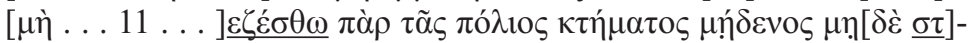

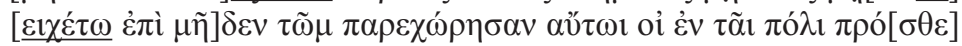

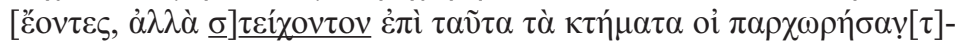

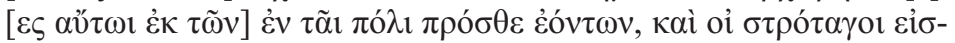

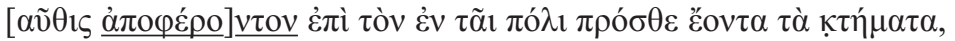

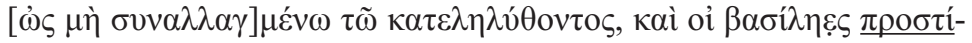

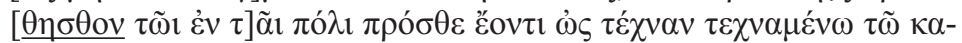

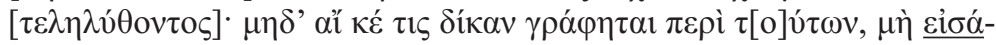

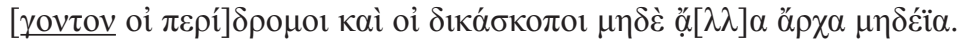

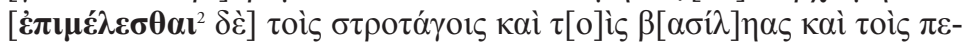

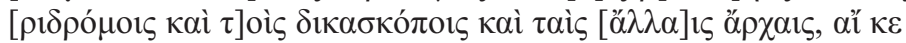

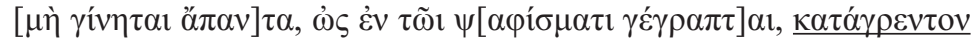

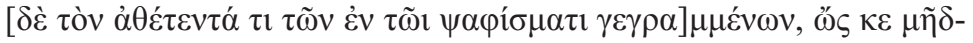

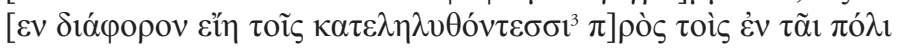

${ }^{2}$ El infinitivo $\dot{\pi} \pi \mu \varepsilon \dot{\lambda} \varepsilon \sigma \theta \alpha \mathrm{l}$ es una conjetura antigua, que se mantiene en todas las reconstrucciones hasta la de Heisserer - Hodot (1986: 125), que la rechazan por razones sintácticas: no se espera de este verbo que tenga como régimen una condicional-interrogativa indirecta como la encabezada por aî $\kappa \varepsilon$ de las ll. 14/15. No obstante, la laguna corresponde al verbo de la frase, que ha de ir en infinitivo,

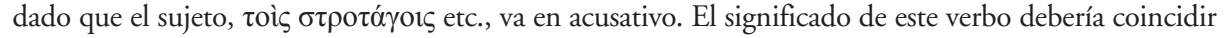
también con la idea de "vigilar", "cuidar de que".

${ }^{3}$ Heisserer - Hodot (1986: 125-126) discuten sobre la reconstrucción de esta laguna, en parti-

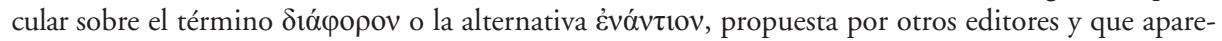
ce en la 1. 24. No proponen, sin embargo, ninguna alternativa, sino que dejan la laguna sin rellenar. El contenido debe corresponder, en todo caso, más a o menos con la conjetura normalmente admitida y que remonta a Blass (1878: 386), que mantenemos. 


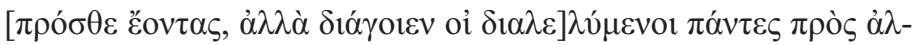

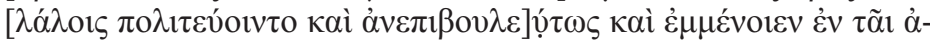

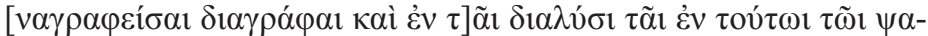

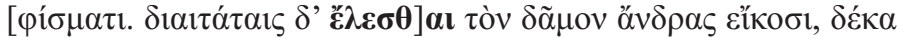

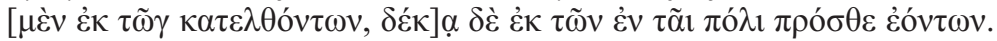

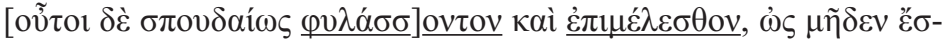

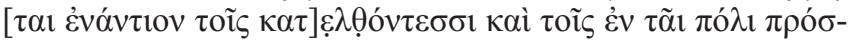

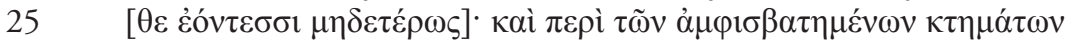

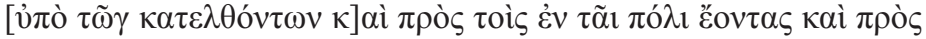

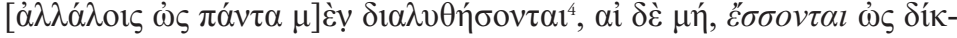

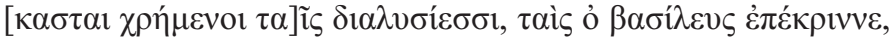

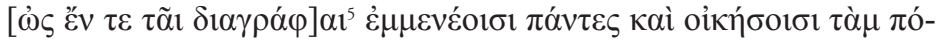

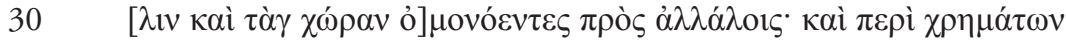

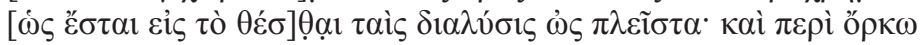

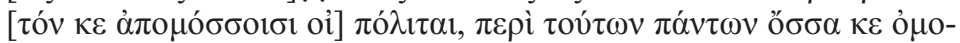

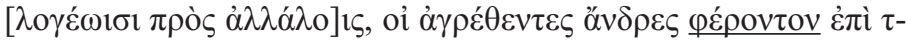

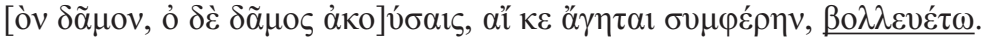

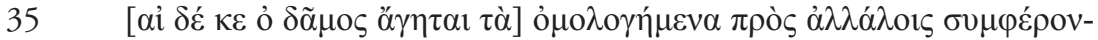

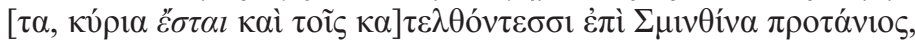

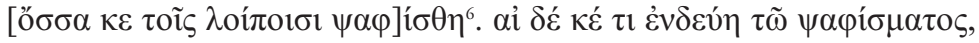

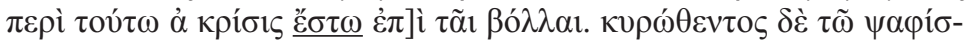

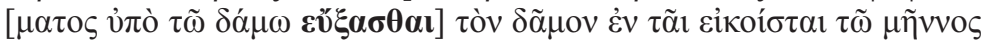
19

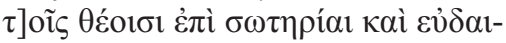

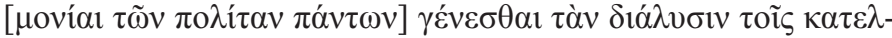

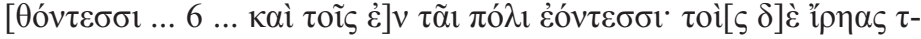

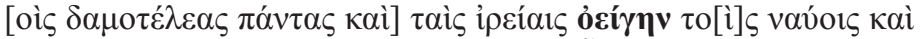

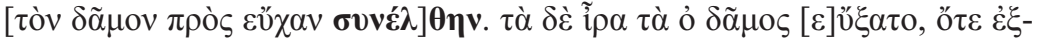

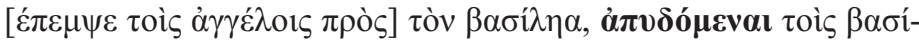

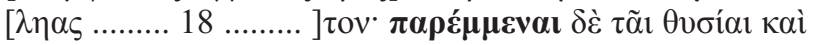

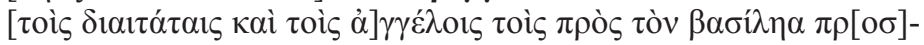

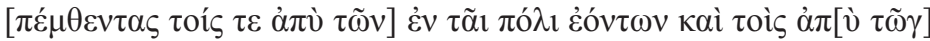

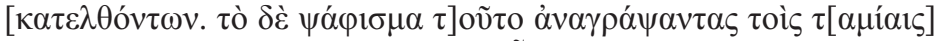

Una posible traducción, a veces tentativa solamente, dadas las lagunas del texto, es la siguiente:

${ }^{4}$ Para su mejor comprensión, incorporamos al texto las conjeturas para las 1l. 26-27 de Heisserer - Hodot (1986: 126), que, sin embargo ellos no llegan a introducir en su edición de la inscripción, sino que dan solo como una posibilidad.

${ }^{5}$ En la reconstrucción de las 11. 28-29 incorporamos al texto las conjeturas de Heisserer - Hodot (1986: 127). Ellos solo las presentan como posibles en su comentario, pero no las incorporan a su edición.

${ }^{6}$ En la reconstrucción de las 11. 36-37 seguimos el mismo proceder que en las líneas a las que se refieren las notas 4 y 5 . 
... que los reyes ${ }^{7}$ den la razón al que ha regresado pues el que estaba en la ciudad antes está tramando un engaño. Pero si uno de los que han regresado no se mantiene en estos términos de la reconciliación ... que no reclame ${ }^{8}$ de parte de la ciudad ningún bien ni tome posesión de nada de lo que le cedieron los que estaban antes en la ciudad, sino que tomen posesión de tales bienes los que se lo cedieron entre los que estaban antes en la ciudad; y que los comandantes devuelvan al que estaba antes en la ciudad los bienes, puesto que el que ha regresado no se ha mantenido en la reconciliación, y que los reyes den la razón al que estaba antes en la ciudad pues que el que ha regresado está tramando un engaño. Y si uno entabla un pleito sobre estas cosas, que no lo admitan los jueces itinerantes ni los supervisores judiciales ni ninguna otra magistratura. Sino que estén atentos los comandantes, los reyes, los jueces itinerantes, los supervisores judiciales y las demás magistraturas si no sucede todo como está recogido en el decreto; y que penalicen al que no haya respetado alguna de las cosas escritas en el decreto, para que no haya ningún diferendo entre los que han regresado y los que estaban antes en la ciudad, sino que en concordia y reconciliados todos unos con otros actúen como ciudadanos y sin sospechas, y que permanezcan en los términos de la estipulación escrita y en la reconciliación recogida en este decreto. Y que elija la asamblea veinte hombres como árbitros, diez de los que han regresado y diez de los que estaban antes en la ciudad. Y que ellos vigilen con atención y se ocupen de que no haya ningún enfrentamiento entre los que han regresado y los que estaban antes en la ciudad. Y con relación a los bienes en litigio por parte de los que han regresado con respecto a los que están en la ciudad y de unos con respecto de los otros, <que se ocupen $>$ de que todo quede reconciliado; y si no, pondrán en ejecución como jueces los términos de la reconciliación que el rey decidió para que se mantengan en lo estipulado y habiten la ciudad y el territorio en concordia unos con otros. Y con relación al dinero, <que se ocupen> de que se impongan los términos de la reconciliación por encima de todo. Y con relación al juramento que hagan los ciudadanos con respecto a todas las cosas cuantas queden juramentadas entre unos y otros, los hombres elegidos deben llevarlo ante la asamblea y la asamblea, tras haber escuchado, si se ve que conviene, que delibere sobre ello; y si la asamblea ve que las cosas acordadas entre unos y otros son convenientes, tendrán validez también para los que han regresado durante la pritanía de Esmitines cuantas cosas se decreten para los demás?. Pero si algo falta todavía en el decreto,

${ }^{7}$ La traducción de los términos técnicos y jurídicos se basa en las propuestas de Heisserer (1980), que recoge y revisa todas las ediciones anteriores. Por motivos de espacio y por no ser este el objetivo de nuestro trabajo, no añadiremos notas adicionales sobre el significado de algunas palabras técnicas, como aquí "reyes", que debe referirse a unos magistrados específicos encargados de la aplicación de decretos de la asamblea.

${ }^{8}$ En IG Suppl. XII 26 (1) se reconstruye $\left.\dot{\varepsilon} \varphi\right] \varepsilon \zeta \dot{\varepsilon} \sigma \theta \omega$, cuyo contenido "apropiarse, tomar posesión” coincide demasiado con $\sigma \tau \varepsilon \chi \chi \varepsilon ́ \tau \omega$, que sigue. Se esperaría algún matiz entre una y otra prescripción.

${ }^{9} \mathrm{La}$ referencia a un período preciso de pritanía muestra que el regreso de los exiliados se estaba produciendo a lo largo de un cierto tiempo. Esta especificación puede entenderse en el sentido de que lo decretado para un momento concreto debería ya quedar como legislado también para situaciones semejantes que se produjeran posteriormente con otros exiliados. 
que la decisión acerca de ello sea en el consejo. Y una vez que quede establecida la vigencia del decreto por parte de la asamblea, que eleve preces toda la asamblea en el día vigésimo del mes ... a los dioses por la salud y la prosperidad de todos los ciudadanos para que haya reconciliación entre los que han regresado y los que están en la ciudad; y que los sacerdotes públicos todos y las sacerdotisas abran los santuarios y que la asamblea se una a las preces. Y las ceremonias sagradas que el pueblo realizó cuando envió a los emisarios hacia el rey que las ofrezcan de nuevo los reyes ... Y que acudan al sacrificio los árbitros y los emisarios enviados al rey de entre los que estaban antes en la ciudad y los que han regresado. Y este decreto, tras haberlo grabado los administradores en una estela de piedra, que lo coloquen en el santuario ...

\section{ANÁLISIS DE LAS ÓRDENES EN ESTE TEXTO}

Este pasaje, según el texto que hemos adoptado, incluye 13 imperativos (subrayados en el texto y la traducción), 8 infinitivos (en negrita) y dos futuros (en cursiva). De ellos, varios han sido reconstruidos total o parcialmente por los diversos editores con más o menos base.

En la mayor parte de los casos hay razones sintácticas que justifican la reconstrucción de la forma. Así, en la línea 1, el imperativo $\pi \rho \circ \sigma \tau i ́ \theta \eta \sigma \tau o v$ se puede reconstruir por el paralelismo entre esta frase y la de las 11. 9-10. El imperativo $\sigma \tau \varepsilon 1 \chi \varepsilon ́ \tau \omega$

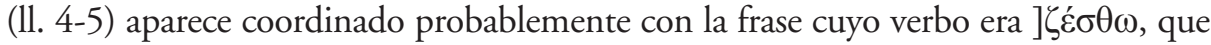
sólo puede ser un imperativo. El imperativo $\pi \rho \circ \sigma \tau 1[\theta \varepsilon \dot{\sigma} \sigma 0 \mathrm{v}$ (1l. 9-10) corresponde al verbo principal de la frase y, dado que su sujeto está en nominativo, el verbo no podría ser un infinitivo. Al principio de la 1.13 falta un verbo, reconstruido por

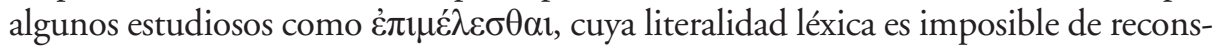
truir, pero que solo puede corresponder a un infinitivo, puesto que su sujeto, $\tau$ oì $\varsigma$

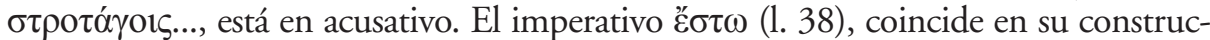
ción con otros imperativos en apódosis de subordinadas condicionales eventuales, como los de 2-5, 11-12 y 34. El infinitivo \&ư $\xi \alpha \sigma \theta \alpha$ (1. 39), de cuya certeza léxica, de nuevo, no podemos estar seguros, corresponde sin duda a una forma de infinitivo,

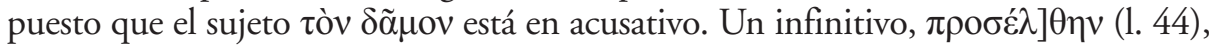
está coordinado con otro infinitivo en la frase anterior, por lo que puede aceptarse como muy probable. Finalmente, cabe reconstruir al final del decreto, siguiendo la fórmula habitual, un infinitivo como $\theta \varepsilon ́ \mu \varepsilon v \alpha r$ en la 1.50.

En definitiva, por tanto, solo queda como dudoso el futuro de la l. 36, ह̌ $\sigma \tau \alpha$, en donde, como verbo de una apódosis eventual, habríamos esperado que fuera, de acuerdo con los otros casos de este decreto, un imperativo. No puede, sin embargo, descartarse que hubiera aquí un futuro. Tomando, por tanto, todas las formas ciertas o probables como base de nuestro trabajo, tendríamos una secuencia de órdenes como sigue (se añaden entre corchetes las reconstruidas total o parcialmente):
1. 1 [Imperativo]
1. 4 Imperativo
11. 4-5 [Imperativo] 


$\begin{array}{ll}\text { 1. } 6 & \text { Imperativo } \\ 1.8 & \text { Imperativo } \\ \text { 11. } 9-10 & \text { [Imperativo] } \\ \text { 11. } 11-12 & \text { [Imperativo] } \\ \text { 1. } 13 & \text { [Infinitivo] } \\ 1.15 & \text { Imperativo } \\ \text { 1. } 21 & \text { Infinitivo } \\ 1.23 & \text { [Imperativo] } \\ \text { 1. } 23 & \text { Imperativo } \\ \text { 1. } 27 & \text { Futuro } \\ 1.33 & \text { Imperativo } \\ 1.34 & \text { Imperativo } \\ 1.36 & \text { [Futuro] } \\ 1.38 & \text { [Imperativo] } \\ 1.39 & \text { [Infinitivo] } \\ 1.43 & \text { Infinitivo } \\ 1.44 & \text { Infinitivo } \\ 1.45 & \text { Infinitivo } \\ 1.46 & \text { Infinitivo } \\ \text { 1. } 50 & \text { [Infinitivo] }\end{array}$

La secuencia alternante, de forma aparentemente irregular, de imperativos e infinitivos, más algún futuro, usados en un mismo texto, de contenido semejante, constituye, como ya dijimos, una base magnífica para tratar de comprobar si las propuestas que ha habido sobre estas formas de transmitir órdenes se confirman o no, buscando un patrón de distribución.

En primer lugar, la propuesta de Delbrück, Wagner y Hentze en sentido de que los imperativos dan órdenes más concretas y los infinitivos órdenes más generales, que no exigen una realización inmediata, no parece poder mantenerse, al menos en este texto. Ciertamente, la instrucción dada desde la línea 13, en la que se reconstruye quizá un infinitivo $\dot{\pi} \pi \mu \varepsilon \dot{\imath} \varepsilon \sigma \theta \alpha 1$ es de carácter muy general, pues instruye en que se respeten las instrucciones dadas en el edicto. Sin embargo, el infinitivo de la línea 21 , č $\lambda \varepsilon \sigma \theta \alpha 1$, transmite una orden muy concreta: que se elijan veinte hombres, diez del grupo de los exiliados y diez del grupo de los que permanecieron en la ciudad.

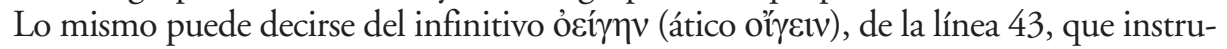

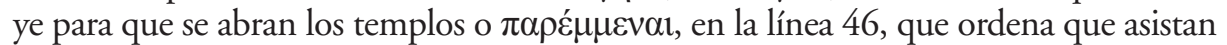
a las ceremonias una serie de personas muy precisas. Por el contrario, los imperativos

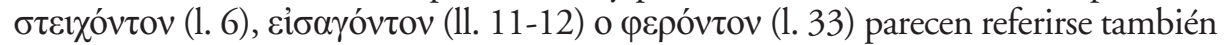
a instrucciones de carácter general, que no están ligadas a un momento inmediato concreto. Por lo tanto, esta no parece la base de la distribución imperativo / infinitivo.

Con respecto a la propuesta de Brioso y otros, solo cabe decir que en este caso no hay ninguna razón para pensar que los infinitivos siguen al imperativo como orden, puesto que, como veremos, lo que sucede es exactamente lo contrario. Por otra parte, el texto analizado en Villa (2018) presentaba exactamente la organización inversa, donde el imperativo cerraba siempre una secuencia de infinitivos.

Por las mismas razones expuestas ante Delbrück, Wagner y Hentze, las propuestas de Hodot y García Ramón no parece que puedan mantenerse, puesto que ambos, 
imperativo e infinitivo, aparecen en contextos bastante semejantes, sin que sea posible establecer una oposición convencionalmente estructuralista entre un término marcado (imperativo) y un término no marcado (infinitivo). Recordemos que muchos de los imperativos, puesto que dependen de situaciones eventuales, no reales, tienen un valor general, que es el que correspondería supuestamente al infinitivo.

Con respecto a la propuesta de Allan, es difícil ver en este texto una diferencia basada en una oposición contextual en la que los imperativos corresponden a órdenes dadas sobre una base legal o consuetudinaria ajena al emisor de la propia orden, frente al infinitivo, que sería una orden dada en términos menos exigentes o perentorios. En este texto todas las instrucciones son producto de las indicaciones recogidas en el edicto y todas deben cumplirse con la misma fuerza. No parece que el emisor de las órdenes esté estableciendo una diferencia sobre el grado de exigencia de las instrucciones dadas.

Finalmente, es preciso atender a la explicación que dan Heisserer - Hodot (1986) con respecto a los datos de esta misma inscripción, pero enmarcados en lo que consideran una forma común de actuación en las inscripciones de Lesbos. Según ellos, el infinitivo se utiliza "para las prescripciones que proceden directamente de la asamblea y que no necesitan deliberación adicional"; el imperativo correspondería a las órdenes relativas a las tareas futuras de los veinte árbitros, que todavía no han sido elegi-

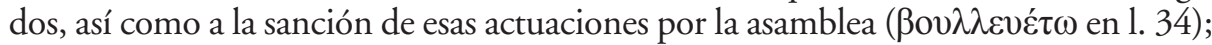
el futuro, por último, se referiría a las consecuencias remotas de los esfuerzos de los árbitros. Sin embargo, eso no parece sostenerse fácilmente: toda la primera serie de órdenes, en las que se indica cómo deben actuar los diferentes magistrados -no solo los veinte árbitros- cuando se produzcan diferendos entre los exiliados y los que se quedaron, están en imperativo y, sin embargo, corresponden a prescripciones generales del decreto y no parece que tengan un origen diferente que el de todas las demás órdenes. En cuanto al único futuro seguro, el de la l. 27, donde se prevé que los árbitros actúen como jueces en determinadas circunstancias, no representa una orden de diferente tipo que la de la 1.23 , donde se les encarga que vigilen y se preocupen de que todo se haga según lo establecido. No parece que el futuro implique consecuencias más remotas que el imperativo en estos casos. En lo relativo al futuro reconstruido en la 1. 36, ya hemos dicho que aquí esperaríamos mejor un imperativo; y es precisamente la eventual identidad de uso, como apódosis de una condicional, de un futuro y un imperativo, lo que demuestra que no hay una diferencia real entre ellos.

Ninguna de las propuestas indicadas, por tanto, parece poder explicar de forma completa y satisfactoria la distribución precisa entre imperativos, infinitivos $\mathrm{y}$ futuros como formas de orden.

\section{UNA PROPUESTA ALTERNATIVA}

Según mi opinión, debemos partir del hecho de que, como defendía también García Ramón, las tres formas alternativas de expresión directiva, imperativo e infinitivo, probablemente se solapaban en gran medida y podían intercambiarse en griego antiguo. Hay múltiples ejemplos de ello en otras lenguas. Lo cual no quiere decir que 
no exista una cierta preferencia para utilizar una forma en un determinado tipo de contexto y la otra forma en otros, como se comprobó en el largo texto de Tesalia estudiado en Villa (2018), donde toda la parte final pasaba al futuro sin explicación aparente, sino solo como una forma de marcar que se trataba de una parte diferente de las anteriores.

En el caso que nos ocupa ahora creo que se puede descubrir una distribución en parte semejante. En primer lugar, si nos fijamos en la distribución de los infinitivos y los imperativos en el edicto que estudiamos, se puede descubrir al menos un rasgo claro: en toda la primera parte del texto predominan los imperativos, mientras que al final, desde la l. 39, solo tenemos infinitivos, conservados o reconstruidos con una cierta verosimilitud. Este último dato, sobre todo, no puede ser fortuito, puesto que manifiesta una voluntad clara de mantenerse en las frases finales en la forma directiva en infinitivo. Por otro lado, también hay infinitivos en toda la primera parte, pero aislados. Sería, por tanto, de esperar que las razones de uso de los infinitivos en un caso y en otro estuvieran relacionadas, al menos dentro del mismo texto. Y, a mi juicio, sí existe ese patrón de distribución. siguientes:

$\mathrm{Si}$ atendemos al contenido del decreto, las partes en que se divide son las

1-12: Qué debe hacerse en cuanto a los problemas que surjan entre los exiliados que han regresado y los ciudadanos que quedaron en la ciudad con relación a los bienes reclamados y, en ocasiones, devueltos a los exiliados.

13-21. Petición general para que se mantenga la concordia entre los exiliados que hayan regresado y los que se habían quedado en la ciudad.

22-30. Han de elegirse veinte hombres, diez de cada uno de los dos grupos, para que diriman las diferencias, que podrán actuar como jueces. El párrafo termina con una referencia muy formal al hecho de que eso es el edicto del rey.

31-38. Cómo deben resolverse las diferencias en lo relativo a los bienes en disputa, al dinero y a los juramentos realizados.

39-49. Sacrificios y ceremonias que deben realizarse.

Si tenemos en cuenta esta distribución, vemos que, quitando el primer apartado, del que no conservamos la primera frase, en los tres siguientes aparece un infinitivo encabezando la instrucción y le siguen luego uno o varios imperativos. El infinitivo parece utilizarse, por tanto, como una forma de marcar cada vez el comienzo de un nuevo tipo de prescripción. Este comienzo no es, sin embargo, más o menos general o más o menos exigente, como ya hemos dicho y como esperaríamos si se cumplieran las previsiones de quienes han estudiado estas formas de orden. Sin embargo, sí parece que es la forma sintáctica que corresponde a lo que podríamos llamar el primer nivel de órdenes.

En efecto, este tipo de decretos están encabezados por una frase formular indicando quién lo ha emitido y a continuación viene la primera instrucción que habitualmente aparece en infinitivo con su sujeto en acusativo, como, por ejemplo, en $I G$ XII 24 [1]:

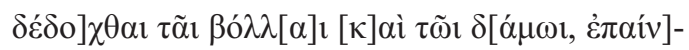

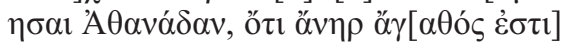

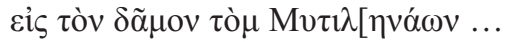


Ha parecido al consejo y a la asamblea ensalzar a Atanadas, ya que es un hombre noble hacia el pueblo de los mitileneos ...

En el caso probable de que el texto que analizamos se presentara más como un edicto de Alejandro, el encabezamiento también requeriría que las primeras instrucciones estuvieran en infinitivo, como en la inscripción que recoge una carta de Alejandro a los ciudadanos de Quíos, contemporánea de la que estudiamos y que comienza del modo siguiente (McCabe, 1986, 32).

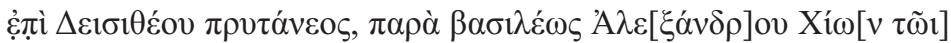 \\ $\delta \eta \dot{\mu} \omega t$. vacat

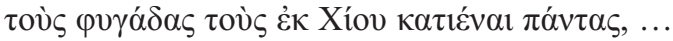

Siendo prítano Disíteo, del rey Alejandro al pueblo de los quiotas: que los exiliados vuelvan todos ...

Una fórmula semejante a cualquiera de las anteriores aparecería en el decreto que nos ocupa. Por lo tanto, la primera instrucción iría seguro en infinitivo. Y después, cada vez que se vuelve a una orden en infinitivo a lo largo del texto, se está recordando por medio de la sintaxis que todo lo que sigue es una instrucción dependiente de la fórmula inicial. En tales condiciones, no es de extrañar que el infinitivo se utilice como marca de comienzo cuando se aborda una nueva serie de órdenes. De esta forma se reasienta la autoridad de las prescripciones. Una vez reasentado este principio, es el imperativo el que va desgranando lo que hay que hacer como consecuencia de la indicación primera.

Al final, una vez establecida la forma en que deben comportarse los exiliados, de cómo deben actuar los magistrados y de cómo debe procederse en caso de querellas, vienen las indicaciones generales de carácter religioso y solemne. Y todas ellas, en la última parte del edicto, aparecen en infinitivo, puesto que se trata de acciones diferentes, no ligadas entre sí, como en el caso de los apartados anteriores.

La interpretación del uso del imperativo y del infinitivo depende, por tanto, de unas circunstancias muy concretas, como ya había indicado Allan (2010). Pero, en este contexto, según me parece, es justo lo contrario de lo que él prevé: al retornar una y otra vez al infinitivo se recupera en cada caso el origen del edicto, dotando a estas prescripciones, precisamente, de más fuerza. Por otra parte, al menos en este caso, los infinitivos sí tienen, como preveían Kühner - Gerth, un carácter enfático, puesto que remiten en cada caso a la fórmula inicial del decreto. Este valor, sin embargo, no tiene por qué estar presente en otras circunstancias, como se veía, por ejemplo, en el texto tesalio estudiado en Villa (2018), donde era el imperativo el que iba estructurando el texto como colofón de cada apartado y adquiría, por tanto, ese valor enfático.

En definitiva, la distribución de las diferentes formas directivas en el documento que estudiamos parece confirmar lo que ya se señaló en Villa (2018): el imperativo y el infinitivo parecen ser en gran medida intercambiables. Sin embargo, se utilizan de un modo contextual, más que general, para establecer una modulación de órdenes en una secuencia de ellas. El contexto determina el valor más enfático o menos de cada uno. En este caso es la sintaxis, dependiente del encabezamiento general, 
hoy perdido, la que hace que el infinitivo remita cada vez al origen del decreto y, por lo tanto, recuerde en cada caso que todo lo dicho en cada apartado emana de él.

\section{UNA OBSERVACIÓN ADICIONAL SOBRE EL FUTURO}

En el texto que estudiamos solo hay una forma segura de futuro, en la 1. 27. Al menos en este caso, su función parece idéntica a la del imperativo. No encabeza ningún apartado temático, sino que solo prevé qué ha de hacerse si alguien no cumple lo establecido. La construcción es en todo semejante a los otros períodos condicionales eventuales ya citados: en todos estos otros casos, tras haber previsto una situación eventual, la frase principal va en imperativo. Nada, por tanto, de un contenido "de esperanza más que de orden" que le atribuyen Kühner - Gerth al futuro yusivo. No puede descartarse, sin embargo, que, fuera de este contexto, en otras situaciones, el futuro pudiera tener ese matiz.

Tampoco parece que, como proponen Heisserer y Hodot, se refiera a las consecuencias remotas de las acciones de los árbitros. Se trata de una prescripción idéntica al resto de las que recoge este decreto.

Todo apunta de nuevo al carácter ampliamente intercambiable de las formas directivas. En este caso el futuro no parece conllevar matiz alguno.

\section{CONCLUSIÓN}

Un largo texto procedente de Mitilene presenta un uso alternante del imperativo, del infinitivo yusivo y, en un solo caso seguro, de un futuro yusivo. El estudio pormenorizado del texto permite identificar un patrón de distribución que no es tanto gramatical, como pragmático, es decir, dependiente del contexto: el infinitivo se utiliza para encabezar párrafos temáticos diferentes; el imperativo se usa para desarrollar las prescripciones de cada párrafo; el futuro, por último, en este texto, se utiliza igual que el imperativo.

Este patrón no coincide de forma plena con las propuestas más importantes realizadas hasta este momento sobre el valor o uso de estas diferentes formas de dar órdenes en griego antiguo. Pero sí parece confirmar algunos aspectos parciales:

1) Las tres formas de dar órdenes parecen haber sido en gran medida intercambiables, tal y como señaló García Ramón (2001).

2) Su uso y distribución es muy dependiente del contexto, como indicó Allan (2010), pero, por ello mismo, parece difícil llegar a proponer un único valor general para cada tipo de orden, como hace él.

3) Coincide el resultado de este trabajo con el de Villa (2018) en el sentido de que el redactor de los textos utiliza las variaciones entre imperativo e infinitivo, sobre todo, para estructurar el texto.

4) En este caso concreto, la construcción con infinitivo seguiría la formulación inicial, hoy perdida, típica de este tipo de texto, y esta fórmula reitera su vigencia cada cierto tiempo precisamente por medio del regreso al infinitivo al comienzo de cada uno de 
los conjuntos temáticos en que se organiza el decreto. En otras palabras, el infinitivo estructura los párrafos del texto.

5) Después de cada infinitivo puede seguir un número variable de imperativos, que se refieren al mismo tipo de previsiones que las que introduce el infinitivo al inicio del párrafo.

6) Al menos en el texto estudiado, el futuro yusivo tiene el mismo uso que el imperativo.

\section{REFERENCIAS BIBLIOGRÁFICAS}

Allan, Rutger (2010): «The infinitivus pro imperativo in Ancient Greek. The Imperatival Infinitive as an Expression of Proper Procedural Action", Mnemosyne 63: 203-228.

BERS, Victor (1984): Greek Poetic Syntax in the Classical Age, Yale University Press, New Haven-Londres. BLASS, Friedrich (1878): «Zu den griechischen Inschriften», Hermes 13: 381-387.

Brioso, Máximo (1990): «Himno Homérico a Hermes 567 ss.: una supuesta laguna», Habis 21: 7-14.

Delbrück, Berthold (1863): De infinitivo Graeco, Inaugural Diss., Ploetz, Halle.

FAZZINI, Erica (2018): «Decreto per gli essuli di Mitilene», Axon 2 (1): 141-160.

GARCía RAMÓN, José Luis (2001): «Impératif et infinitif pro imperatiuo dans les textes grecs dialectaux : les lois de Gortyne», en R. HODOT (ed.), Les modes dans les dialectes grecs anciens (Verbum 23.3), Presses de l'université de Nancy, Nancy, pp. 341-360.

HEISSERER, Andrew J. (1980): Alexander the Great and the Greeks. The epigraphic evidence, University of Oklahoma Press, Norman.

HeIsSERER, Andrew J. - Hodot, René (1986): «The Mytilinean Decree of Concord», ZPE 63: 109-128.

Hentze, Carl (1902): «Der imperativische Infinitiv in den homerischen Gedichten», Beiträge zur Kunde der indogermanischen Sprachen 27: 106-137.

Hicks, Edward L. - HiLl, George F. $\left(1901^{2}\right)$ : A Manual of Greek Historical Inscriptions, Clarendon Press, Oxford.

Hodot, René (1976): «Notes critiques sur le corpus épigraphique de Lesbos», EAC 5: 17-91.

KÜHNER, Raphael - GERTH, Bernhard (1898-1904): Ausfïhrliche Grammatik der griechischen Sprache, vol. II (Satzlehre I-II), Hahn, Hannover - Leipzig.

MCCABE, Donald F. (1986): Chios Inscriptions. Text and List, James V. Brownson Institute for Advanced Studies, Princeton.

Neuberger-Donath, Ruth (1980): «The obligative infinitive in Homer and its relationship to the imperative», Folia Linguistica 14: 65-82.

DE LA VILLA, Jesús (2018): «More on Aspect and Modality in Thessalian Official Documents», en G. Giannakis et al. (eds.) Studies in Ancient Greek Dialects. From Central Greece to the Black Sea, De Gruyter, Berlín, pp. 405-428.

WACKERNAGEL, Jacob $\left(1950^{2}\right)$ : Vorlesungen über Syntax, Birkhäuser, Basilea.

WaGneR, R. (1891): Der Gebrauch des imperativischen Infinitivs in den homerischen Gedichten. Programm, Grossherzogliches Gymnasium Fridericianus, Schwerin. 
